\title{
Tungsten erosion at the ICRH limiters in ASDEX Upgrade
}

\author{
R. Dux ${ }^{\text {a }}$, V. Bobkov ${ }^{\text {a }}$, N. Fedorczak ${ }^{\mathrm{b}}$, K. Iraschko ${ }^{\mathrm{a}}$, \\ A. Kallenbach ${ }^{\text {a }}$, R. Neu ${ }^{\text {a }}$, T. Pütterich ${ }^{\text {a }}$, V. Rohde ${ }^{\text {a }}$, \\ ASDEX Upgrade Team ${ }^{\text {a }}$ \\ ${ }^{a}$ Max-Planck-Institut für Plasmaphysik, EURATOM Ass., D-85748 Garching, Germany \\ ${ }^{\mathrm{b}}$ École Normale Supérieure, Département de Physique, Paris, France.
}

\begin{abstract}
All limiters on the low field side of ASDEX Upgrade are equipped with tungsten coated tiles. The $\mathrm{W}$ influx from the limiters was measured with a new spectroscopic setup providing sufficient time resolution to resolve the influx peak during edge localised modes (ELM). The ELM peak is mainly due to an increase of the mean ion energy hitting the $\mathrm{W}$-surface. W influx due to sputtering by fast $D^{+}$ions from neutral beam injection was measured for different injection angles and compared to Monte Carlo calculations of the fast ion loss. The erosion depends less strongly on the injection angle than predicted by the code due to an enhancement of the fast ion transport by ELMs. At the antenna limiters of the ion cyclotron resonance heating (ICRH), the influx rapidly increases when the ICRH is switched on, which can be explained by a rise of the average sheath potential at the limiter.
\end{abstract}

Key words: First wall materials Tungsten, Erosion, ASDEX Upgrade

PACS: 52.40.Hf, 52.25.Vy, 52.55.Fa

Corresponding author: Ralp Dux, Boltzmannstr.2, D-85748 Garching, Germany.

E-mail address: Ralph.Dux@ipp.mpg.de

Tel.+49-89-3299-1256;

fax:+49-89-3299-1812

\section{Introduction}

In order to test the reactor compatibility of high-Z plasma facing components (PFC), a step-bystep increase of tungsten coated PFCs towards a full tungsten machine is pursued at ASDEX Upgrade. At present, about $85 \%$ of the total PFC area consists of W-coated graphite tiles [1]. 
The last enhancements concentrated on the most critical PFCs receiving the highest particle and energy flux densities in the main chamber: the poloidal limiters of the 4 ICRH antennas, which are a part of the window frame surrounding each antenna, and the guard limiters, which are located at each side of the 2 neutral beam ducts. The main reasons for the high flux densities onto these limiters are the relatively small wetted area, which is approximately a factor of 30 smaller than the area of the heat shield at the inner column, the small distance to the separatrix, and the predominance of fast particle losses at the low field side of the tokamak. The coating of these limiters has now been completed after initial tests in 2003-2005, where at first, one guard limiter [2] and subsequently, one ICRH limiter was equipped with W-coated test tiles.

From the operation in 2005, it became evident that fast particles from neutral beam injection (NBI) as well as impurity ions accelerated in the rectified sheath in front of the ICRH antennae play an important role for the $\mathrm{W}$ erosion [3] at the limiters, while CX neutrals yield only a minor contribution [4]. This paper discusses further details of the different sputtering contributions, which could be resolved due to a significant increase in the time resolution of the spectroscopic setup. Further details on ICRH induced W-erosion can be found in [5]. ICRH induced Moerosion was studied in Alcator C-Mod [6].

\section{Experimental Setup and Influx Determination}

Three tungsten limiters are observed by optical heads which are located behind the tiles of the inner column. Fifteen lines-of-sight are used to measure influx profiles along the limiter height. Each line-of-sight has a diameter of $\approx 2.5 \mathrm{~cm}$ and is toroidally oriented on that part of the limiter, which has minimum distance to the plasma. The light is transfered via optical fibres to the entrance slit of a $\mathrm{f}=180 \mathrm{~mm}$ spectrometer with high f-number of 2.8 . The spectrum $(\lambda=398.5-414.5 \mathrm{~nm})$ of all fibres is measured by a back-illuminated frame-transfer CCD camera. Compared to previous campaigns, the new spectroscopic setup has strongly increased the available time resolution from $70 \mathrm{~ms}$ to $\approx 3 \mathrm{~ms}$ for simultaneous measurement on all channels and $253 \mu$ s for measurements on a single line-of-sight. Tungsten influx is monitored by measuring WI line radiation at $400.8 \mathrm{~nm}$, while the Balmer- $\delta$ transition at $410.1 \mathrm{~nm}$ is used to calculate the deuterium influx. The measured photon fluxes are transformed into ion fluxes using the number of ionisations per emitted photon, i. e. the $(S / X B)$ value. For tungsten $(S / X B)=20$ is used [7], and for $\mathrm{H}_{\delta}$, the atomic value $(S / X B)=3.3 \times 10^{3}$ is multiplied by a factor of 1.5 as a rough estimate for the molecular flux contribution [8]. The heavy tungsten atoms radiate very close to the limiter and the spectroscopic measurement yields the local erosion flux at the observed spot. Deuterium, which starts inside the spot, can travel a longer path before being ionised and the photon emitting deuterium cloud was measured to be about $5 \mathrm{~cm}$ wide [2]. Thus, the deuterium flux density is multiplied by a factor of 2 , i. e. the ratio of the widths of the emission cloud and 


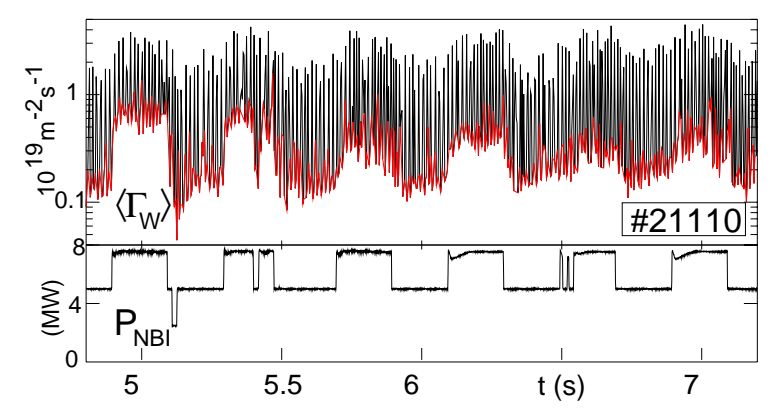

Fig. 1. Spatially averaged tungsten influx from an ICRH limiter during a scan of different NBI sources added to a steady $5 \mathrm{MW}$ NBI heating $\left(f_{E L M}=76 \mathrm{~Hz}, \Delta R_{\text {sep }}=4 \mathrm{~cm}\right)$. The radial beams are followed by the tangential beams.

the spot size, where poloidal homogeneity of the hydrogen influx on the $5 \mathrm{~cm}$ scale is assumed, such that the poloidal dimension of the emission cloud drops out.

Erosion yields are calculated by dividing the $\mathrm{W}$ flux density $\Gamma_{W}$ by the deuterium flux density $\Gamma_{D}$. The quantity $Y_{e f f}=\Gamma_{W} / \Gamma_{D}$ is an effective yield. It includes the sputtering by deuterium as well as by plasma impurities. The interpretation of $Y_{\text {eff }}$ is based on the physical sputtering of tungsten $[9,10]$. Thermal plasma ions have a kinetic ion energy at the wall of roughly $(2+$ $3 Z) k_{B} T$. Considering an admixture of $1 \% \mathrm{C}^{4+}$ to the ion flux of $\mathrm{D}^{+}, Y_{\text {eff }}$ is dominated by carbon sputtering and is $10^{-4}$ at $6.6 \mathrm{eV}$ and $10^{-3}$ at $28 \mathrm{eV}$. Sputtering by pure $\mathrm{D}^{+}$ion flux reaches $10^{-4}$ at $54 \mathrm{eV}$. Thus, only $\mathrm{D}$ sputtering by fast ions is relevant. For D sputtering, $Y$ has a broad maximum of $\approx 10^{-2}$ at $E=6 \mathrm{keV}$ with $Y>8 \times 10^{-3}$ for $E=2-28 \mathrm{keV}$.

\section{Tungsten influx due to fast NBI ions}

A fraction of the fast $\mathrm{D}^{+}$ions from the neutral beam injection (NBI) heating is transported onto the limiters during the slowing down process and causes sputtering of tungsten. The loss probability for a fast ion increases with decreasing radial distance to the plasma edge and decreasing velocity fraction along the magnetic field direction $\left(v_{\|} / v\right)$, i.e. increasing pitch angle. The eight beam sources at ASDEX Upgrade have different beam geometries, and the fast ions are born with an average pitch angle of $\left\langle v_{\|} / v\right\rangle \approx 0.2$ for the most radial beam and $\left\langle v_{\|} / v\right\rangle \approx 0.7$ for the most tangential beam.

Type-I ELMy H-mode discharges with constant heating by 2 NBI sources $\left(\left\langle v_{\|} / v\right\rangle \approx 0.46\right)$ were performed, where each $400 \mathrm{~ms}$ one of the remaining 6 sources was added for a duration of $200 \mathrm{~ms}$. Fig. 1 shows the variation of the average tungsten influx $\left\langle\Gamma_{W}\right\rangle$ on all six channels of one ICRH limiter during the scan of the additional beam sources, which starts with the most radial beam and ends with the most tangential beam. The rapid fluctuations are due to the type-I ELMs which had a frequency $f_{E L M}=76 \mathrm{~Hz}$. The scans were repeated at two distances between 


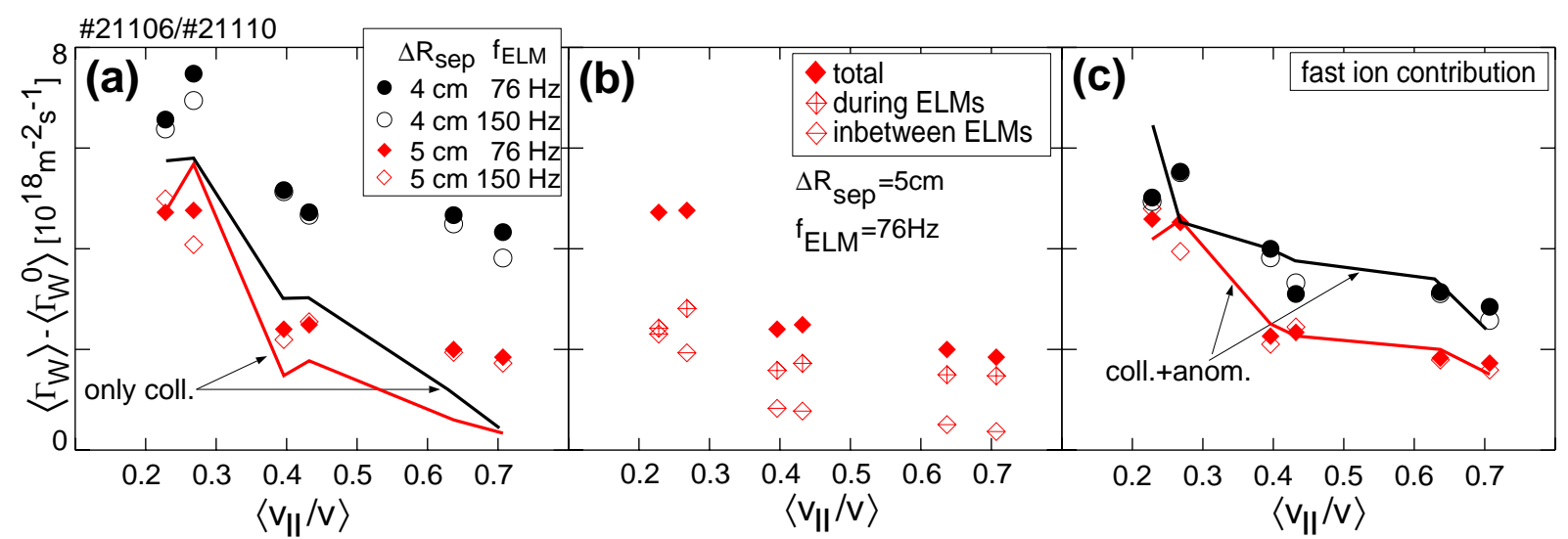

Fig. 2. In (a), spatially and temporally averaged change of tungsten influx is shown versus $\left\langle v_{\|} / v\right\rangle$ of each additional NBI source for two ELM frequencies and two distances between separatrix and limiter. The lines give the modeling results with pure collisional transport. In (b), the different contributions in-between and during ELMs are displayed for a low ELM frequency case. In (c), the estimated pure fast ion contribution is shown and compared to the model including anomalous fast ion transport by ELMs.

separatrix and limiter of $\Delta R_{\text {sep }}=4 \mathrm{~cm}$ and $5 \mathrm{~cm}$ and at a higher deuterium puff level, which increased the ELM frequency to $150 \mathrm{~Hz}$. The sputtering caused by each source was evaluated by taking the difference of the influx densities with and without the additional beam. Fig.2(a) displays the temporal mean of these differences $\left\langle\Gamma_{W}\right\rangle-\left\langle\Gamma_{W}^{0}\right\rangle$ versus $\left\langle v_{\|} / v\right\rangle$ of each beam. The limiter $\mathrm{W}$-erosion is clearly enhanced for the more radial beams compared to the tangential beams.

Code calculations have been performed to model the fast particle load and the respective tungsten erosion rates at the limiters in detail. The start position and velocities of the fast NBI ions were calculated with the Monte Carlo code FAFNER [11]. Subsequently, the orbit of the gyro centre of the ions was calculated including pitch angle scattering and slowing down collisions. The magnetic field components were taken from the equilibrium reconstruction and also the toroidal magnetic field ripple was included in the calculation. Each particle is followed until the particle energy drops below the local value of $3 k_{B} T_{i}$ or until it hits the contour of a limiter, the inner heat shield, or the divertors. A hit with a surface contour is obtained in the code, if the particle is toroidally in front of the contour and if the part of the Larmor circle, which was covered during the last time step, intersects the contour. For the collision frequencies, density and temperature profiles are taken from the experiment. Each calculation starts with about 10000 ions to obtain the fast ion load. From the energy of the hitting particle, the sputtering yield is calculated, which finally gives the total erosion rate by fast NBI ions when normalising to the total NBI power.

The modeling results are overlaid as lines in Fig.2(a), which predict a much stronger decay 
of the limiter erosion with increasing $\left\langle v_{\|} / v\right\rangle$ than experimentally observed. The weaker experimental dependence on $\left\langle v_{\|} / v\right\rangle$ is due to the transport induced by ELMs, which could be temporally resolved in the low frequency case. In Fig. 1 the lower influx envelope (marked red) shows a much stronger dependence on the beam source than the ELM peaks. In Fig.2(b), the different contributions during and in-between ELMs are separately displayed. In the high ELM frequency case, the spectroscopic time resolution is not sufficient to resolve the ELMs, however, the time averaged signals in Fig.2(a) are identical in the two cases. Thus, the effect of each ELM on the W erosion decreases with increasing ELM frequency as is usually observed for the loss of plasma energy. The ELM is expected to cause a limiter load in the fast ion channel as well as in the thermal channel. The latter can not be calculated directly since there are no direct measurements of the temperature increases at the limiter and the following estimate of the thermal contribution shall only present a consistent picture. The fluxes of boron and carbon were measured using multiplets of BII at $412.3 \mathrm{~nm}$ and of CII at $514.3 \mathrm{~nm}$, which increased with each beam but showed no dependence on the injection angle. The thermal sputtering due to $\mathrm{B}$ and $\mathrm{C}$ was calculated using an average temperature of $5 \mathrm{eV}$ for $\Delta R_{\text {sep }}=5 \mathrm{~cm}$ and $9 \mathrm{eV}$ for $\Delta R_{\text {sep }}=4 \mathrm{~cm}$. Thus, the pure fast ion contribution to the $\mathrm{W}$ erosion was estimated, which is shown in Fig.2(c). The effect of ELMs on the fast ion loss is treated in the code with ad hoc assumptions, which need to be replaced by a more physical description in the future. An anomalous pitch angle scattering frequency is added to the collisional value as a first attempt to include the ELM transport into the model. The additional frequency has its maximum value of $\nu=100 \mathrm{~s}^{-1}$ a few $\mathrm{cm}$ inside the separatrix and decays towards the inside and the outside. At this maximum position, the collisional frequency is $5-50 \mathrm{~s}^{-1}$ for ion energies $20-90 \mathrm{keV}$ respectively. This choice yields the fit with the data given by the lines in Fig.2(c). Besides the fit of the average limiter fluxes, the calculations are also in good agreement with the measured spatial profiles on the ICRH limiter and the guard limiter, which differ due to differences in the position and shape of the limiters.

\section{ICRH induced tungsten influx}

When ICRH heating is switched on/off, the tungsten influx at the limiters of the active ICRH antennas strongly increases/decreases with a rise/decay time below $1 \mathrm{~ms}$. The increase is strongest at the active antenna but also visible at the neighbouring guard limiter at a toroidal distance of $\approx 0.8 \mathrm{~m}$ and at the opposite ICRH limiter at a distance of $\approx 5 \mathrm{~m}$ [3]. The ICRH contribution to the total limiter influx was estimated by comparing ICRH on/off phases during a number of discharges. The fraction due to ICRH rises from 60 to $90 \%$ on average with increasing ICRH power fraction varying from 12 to $65 \%$, respectively.

The fast temporal response and the spatial structure rules out a predominant contribution of ICRH induced fast ions to the $\mathrm{W}$ erosion and points towards the sheath rectification effect as 


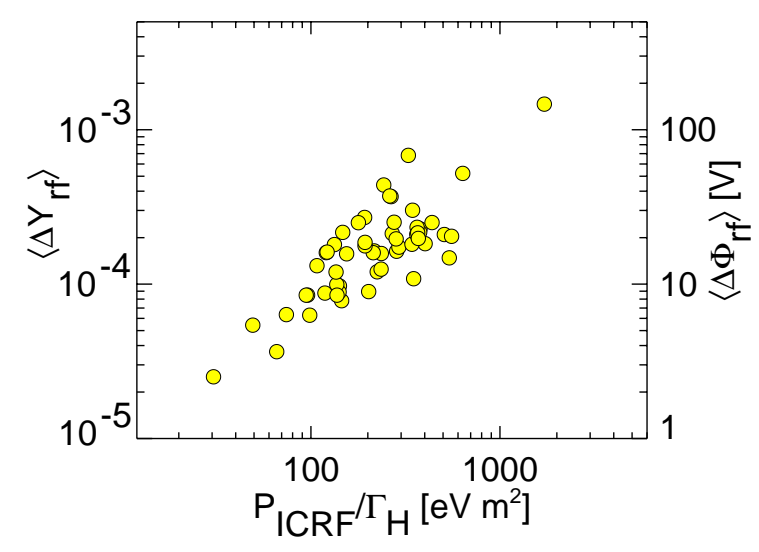

Fig. 3. Mean difference of the effective yields $\left\langle\Delta Y_{r f}\right\rangle$ and according increase of the sheath potential $\left\langle\Delta \Phi_{r f}\right\rangle$ at the ICRH limiter versus the ratio of ICRH power and hydrogen recycling flux density $P_{I C R H} / \Gamma_{H}$.

main reason to the increased $\mathrm{W}$ influx. The additional average sheath potential $\Delta \Phi_{r f}$ accelerates the sputtering ion onto the target which leads to an increased erosion yield. For a $\mathrm{D}^{+}$plasma with an admixture of $1 \% \mathrm{C}^{4+}$, synonymous with the mixture of light impurity ions, the difference of the effective yields $\Delta Y_{r f}$ with and without additional sheath potential is only weakly dependent on the edge temperature and for $\Delta \Phi_{r f}$ between $1 \mathrm{~V}$ and $1 \mathrm{kV}$, it is roughly $\Delta \Phi_{r f}[\mathrm{~V}]=10^{5} \Delta Y_{r f}$. $\Delta Y_{r f}$ can be determined from consecutive time slices with/without ICRH during a discharge and serves as a simple estimate of the sheath potential increase. For H-mode discharges with type-I ELMs heated by NBI and ICRH, Fig. 3 shows the mean sheath potential increases at the ICRH limiter, which approximately fill the range $1-100 \mathrm{~V}$. The data set is shown versus $P_{I C R H} / \Gamma_{H}$ since $\Delta \Phi_{r f}$ increases with ICRH power and decreases with the hydrogen recycling level. The uncertainty of $\Delta Y_{r f}$ is estimated to be a factor of 4 .

\section{Effect of ELMs}

A few discharges were measured with high time resolution of $253 \mu$ s on a single line-of-sight to resolve single type-I ELMs. The ELM frequency varied between 46 to $175 \mathrm{~Hz}$ and the ELM energy $W_{E L M}$ decreased from 25 to $6.7 \mathrm{~kJ}$ with an approximate $1 / f_{E L M}$ dependence. Each ELM causes a drastic increase of the tungsten influx by more than an order of magnitude. In Fig.4(a), the tungsten fluence during an ELM is seen to rise approximately linear with $W_{E L M}$ for a set of purely NBI heated plasmas (5-7.5 MW) and with a 3 times larger slope for a set with additional ICRH heating of $0.7 \mathrm{MW}$ per antenna. The increased $\mathrm{W}$ influx during ELMs is mainly due to a strong increase of the effective sputtering yield as can be seen in Fig.4(b). Here, $Y_{\text {eff }}$ is shown for the phases inbetween ELMs and during ELMs. The ELM values are a temporal average during the whole ELM, i.e. the ratio of the tungsten and hydrogen fluences. $Y_{\text {eff }}$ rises by about a factor of 10 during the ELMs for pure NBI heating and less for the few 


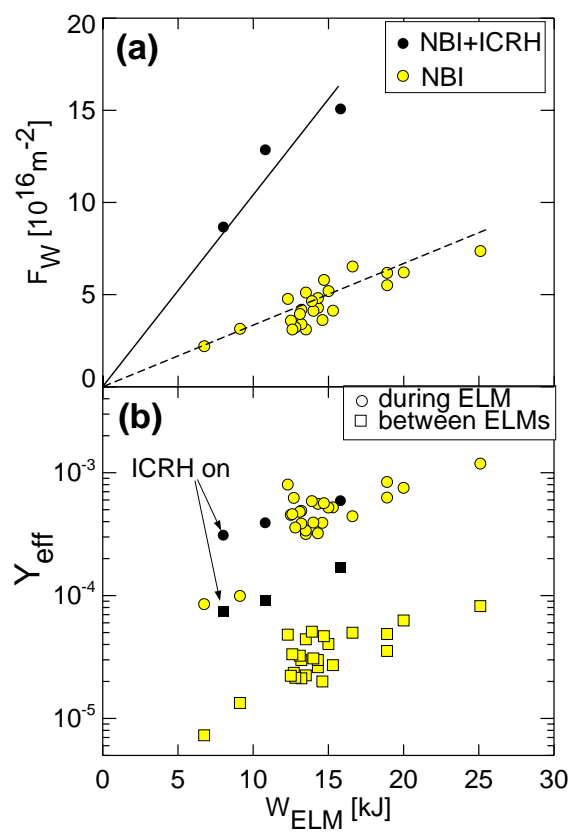

Fig. 4. Fig.(a) shows the tungsten fluence $F_{W}$ during type-I ELMs in the middle of an ICRH limiter versus the ELM energy loss of the plasma $W_{E L M}$ for discharges with NBI heating and additional ICRH heating. For the same data set, Fig.(b) depicts the effective tungsten yield during and in-between ELMs. points with ICRH (ICRH does not switch off during ELMs), which have higher $Y_{\text {eff }}$ between ELMs. This rise reflects an increased mean energy of the ions hitting the $\mathrm{W}$-surface, which is probably due to an increase of the edge temperature as well as an increase of the fast ion load onto the limiters. For the whole data set including the ICRH points, the fraction of the $\mathrm{W}$ fluence during ELMs to the total fluence during and inbetween ELMs does not dependend on the ELM size and is $(70 \pm 10) \%$. It has to be noted, that these data give only the local behaviour at one position in the middle of the ICRH limiter and not the changes of the global influx from all limiters. The effect of ELMs was also measured for the influx of the low-Z elements boron and carbon. The influx during ELMs changes much less and the ELM contribution to the total influx is only $45 \pm 5 \%$.

\section{Conclusion}

The tungsten erosion at the low field side limiters in ASDEX Upgrade was spectroscopically measured. In diverted discharges, $\mathrm{W}$ influx densities are only detectable when NBI or ICRH are used. NBI leads to a sputtering by fast $\mathrm{D}^{+}$ions. The fast ion loss is enhanced by ELMs. Code calculation of the fast ion sputtering are in good agreement with the measurements. ICRH leads to an enhancement of the erosion yields from sheath accelerated impurity ions, which is probaly due to a rise of the sheath potential. The sheath potential increases were estimated from the increase of the effective yield and vary between 1-100 V in H-mode discharges. This effect 
makes the ICRH to account for $60-90 \%$ of the W influx from the limiters. In H-modes with type-I ELMs, about $70 \%$ of the local W-influx at the middle of ICRH limiter appears during ELMs. During the ELM the mean energy of the ions hitting the W-surface is increased, which is the predominant contribution to the rise of the tungsten influx.

\section{References}

[1] R. Neu et al., PSI 2006, Hefei, submitted to J. Nucl. Mater. (2006).

[2] R. Dux, A. Herrmann, A. Kallenbach, et al., J. Nucl. Mater. 337-339 (2005) 852.

[3] R. Dux, et al., 32nd EPS Conference, Tarragona, vol. 29C, pages P-1.010, Geneva, 2005, EPS.

[4] A. Kallenbach, R. Neu, R. Dux, et al., Plasma Phys. Controlled Fusion 47 (2005) B207.

[5] V. Bobkov et al., PSI 2006, Hefei, submitted to J. Nucl. Mater. (2006).

[6] B. Lipschultz et al., PSI 2006, Hefei, submitted to J. Nucl. Mater. (2006).

[7] A. Thoma, K. Asmussen, R. Dux, et al., Plasma Phys. Controlled Fusion 39 (1997) 1487.

[8] H. P. Summers, Tech. Report JET-IR 06, JET Joint Undertaking, Abingdon, UK, 1994.

[9] W. Eckstein, et al., Tech. Report IPP 9/82, MPI für Plasmaphysik, Garching, Germany, 1993.

[10] W. Eckstein, Tech. Report IPP 9/132, MPI für Plasmaphysik, Garching, Germany, 2002.

[11] G. Lister, Tech. Report IPP 4/222, MPI für Plasmaphysik, Garching, Germany, 1985. 